\title{
A Nonaveraging Set of Integers With a Large Sum of Reciprocals
}

\section{By J. Wróblewski}

\begin{abstract}
A set of integers is constructed with no three elements in arithmetic progression and with a rather large sum of reciprocals.
\end{abstract}

It is a famous open question due to Erdös [2] whether every infinite sequence of positive integers $a_{i}(i=1,2, \ldots)$ such that

$$
\sum_{i=1}^{\infty} \frac{1}{a_{i}}=\infty
$$

contains arbitrarily long arithmetic progessions. It is not even known whether there exist sequences $a_{i}$ containing no three terms in arithmetic progression (called for the sake of brevity nonaveraging sets) such that the sum $\sum_{i=1}^{\infty} 1 / a_{i}$ is arbitrarily large.

Gerver (see [4]) constructed sequences containing no $k$-term arithmetic progression with the sum of reciprocals greater than $(1-\varepsilon) k \cdot \log k$, where any $\varepsilon>0$ is appropriate for all but a finite number of integers $k \geqslant 3$.

A well known nonaveraging set apparently first studied by G. Szekeres (see [3]), consists of the numbers $1+3^{\alpha_{1}}+3^{\alpha_{2}}+\cdots+3^{\alpha_{k}}$, where $k \geqslant 0$ and $0 \leqslant \alpha_{1}<\alpha_{2}$ $<\cdots<\alpha_{k}$. Denoting it by $S$ we have

$$
3.00793<\sum_{a \in S} \frac{1}{a}<3.00794
$$

(cf. [5] where the value of the sum is given as 3.007).

The aim of this note is to construct a nonaveraging set of integers with the sum of reciprocals appreciably greater than $\sum_{a \in S} 1 / a$. The construction uses the idea of Behrend [1]. Let, for $p, q>0, r \geqslant 0, B(p, q, r)$ be the set of all integers of the form

$$
\sum_{i=1}^{q} k_{i}(2 p-1)^{i-1}
$$

where $0 \leqslant k_{i}<p$ for $i=1,2, \ldots, q$ and $\sum_{i=1}^{q} T_{p}\left(k_{i}\right)=r$ with

$$
T_{p}(k)=\frac{(k-[(p+1) / 2]) \cdot(k-[(p-1) / 2])}{2} .
$$

Lemma 1. The set $B(p, q, r)$ is nonaveraging. Moreover, for $s \in B(p, q, r)$ we have $0 \leqslant s<\frac{1}{2}(2 p-1)^{q}$. 
The proof is similar to that of Behrend [1] whose $k_{i}^{2}$ has been replaced here by $T_{p}\left(k_{i}\right)$.

Let $Z, T$ be two finite sets of nonnegative integers, and let $z, t$ be the greatest elements of $Z, T$ respectively. Define $(Z, T)$ by the formula

$$
(Z, T)=Z \cup T+m+z+1 \cup T+3 m+2 t+z+3 \cup T+3 m+4 t+z+4,
$$

where $m=\max (z, t)$ and $T+x=\{a+x: a \in T\}$.

LEMMA 2. If $Z, T$ are nonaveraging, so is $(Z, T)$.

The proof is by straightforward verification.

Now we give the construction of our set. Put

$$
\begin{aligned}
& Z_{0}=\{n \in S: n \leqslant 21523361\} \\
& Z_{1}=\left(Z_{0}, B(4,9,5)\right), \quad Z_{2}=\left(Z_{1}, B(4,10,5)\right)
\end{aligned}
$$

and let for $n \geqslant 3$

$$
Z_{n}=\left(Z_{n-1}, B\left(6, n+6, r_{n}\right)\right), \quad \text { where } r_{n}= \begin{cases}{\left[\frac{4(n+6)}{3}\right]} & \text { for } n \neq 5 \\ 15 & \text { for } n=5\end{cases}
$$

By definition $Z_{0} \subset Z_{1} \subset Z_{2} \subset \cdots$ and Lemmas 1 and 2 imply that the set $Z=\bigcup_{n=0}^{\infty} Z_{n}$ is nonaveraging.

Computation performed on the computer ODRA 1305 of the Wrockaw University shows that $\sum_{a \in Z} 1 / a>3.00849$. Thus we have established

THEOREM. There exists a nonaveraging set of integers with the sum of reciprocals greater than 3.00849 .

ul. Leczycka $11 / 3$

53-632 Wroclaw, Poland

1. F. Behrend, "On sets of integers which contain no three terms in an arithmetic progression," Proc. Nat. Acad. Sci. U.S. A., v. 32, 1946, pp. 331-332.

2. P. ERDös, "Problems and results in combinatorial number theory," Astérisque, v. 24-25, 1975, pp. 295-310.

3. P. ERdös \& P. TURÁN, “On some sequences of integers,” J. London Math. Soc., v. 11, 1936, pp. 261-264.

4. J. Gerver, "The sum of the reciprocals of a set of integers with no arithmetic progression of $k$ terms," Proc. Amer. Math. Soc., v. 62, 1977, pp. 211-214.

5. J. GeRVER \& L. RAMSEY, "Sets of integers with no long arithmetic progressions generated by the greedy algorithm,” Math. Comp., v. 33, 1979, pp. 1353-1360. 